\begin{tabular}{|c|c|c|c|c|c|c|}
\hline \multirow{4}{*}{ Impact Factor: } & ISRA (India) & $=3.117$ & SIS (USA) & $=0.912$ & ICV (Poland) & $=6.630$ \\
\hline & ISI (Dubai, UAE & $=0.829$ & РИНЦ (Russia) & $=0.156$ & PIF (India) & $=1.940$ \\
\hline & GIF (Australia) & $=0.564$ & ESJI (KZ) & $=8.716$ & IBI (India) & $=4.260$ \\
\hline & JIF & $=1.500$ & SJIF (Morocco) & $=5.667$ & OAJI (USA) & $=0.350$ \\
\hline
\end{tabular}

\section{SOI: $1.1 /$ TAS $\quad$ DOI: $10.15863 /$ TAS International Scientific Journal Theoretical \& Applied Science}

p-ISSN: 2308-4944 (print) e-ISSN: 2409-0085 (online)

Year: $2019 \quad$ Issue: $05 \quad$ Volume: 73

Published: $10.05 .2019 \quad$ http://T-Science.org
QR - Issue

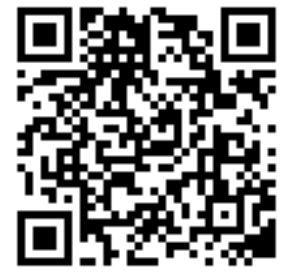

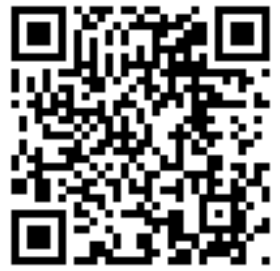

Unona Krahmaleva

Candidate of Science

Taraz State University named M.H.Dulaty

Vyacheslav Shevtsov graduate student of the 2 nd course of the specialty "Mathematics»

Taraz State University named M.H.Dulaty

\title{
THE HOMOGENEOUS SOLUTION OF PROBLEMS OF MATHEMATICAL PHYSICS IN THE MAPLE ENVIRONMENT
}

\begin{abstract}
One of the most common and effective methods of solving homogeneous problems of mathematical physics is the Fourier method or the method of separating variables. As you know, the main idea of this method is that the solution of the initial problem is reduced to the solution of auxiliary problems for equations with fewer independent variables. In particular, if the given equation contains 2 independent variables, the auxiliary problems will be dependent on one variable.

Key words: Fourier method, Maple, differential equation.

Language: Russian

Citation: Krahmaleva, U., \& Shevtsov, V. (2019). The homogeneous solution of problems of mathematical physics in the maple environment. ISJ Theoretical \& Applied Science, 05 (73), 401-406.

Soi: http://s-o-i.org/1.1/TAS-05-73-59 Doi: crossef https://dx.doi.org/10.15863/TAS.2019.05.73.59

\section{РЕШЕНИЕ ОДНОРОДНЫХ ЗАДАЧ МАТЕМАТИЧЕСКОЙ ФИЗИКИ В СРЕДЕ МАРLЕ}

Аннотация: Один из распространенных и эффективных методов решения однородных задач математической физики - метод Фурье или метод разделения переменных. Как известно, основная идея этого метода состоит в том, что решение исходной задачи сводится к решению вспомогательных задач для уравнений с меньшим числом независимых переменных. В частности, если заданное уравнение содержит 2 независимые переменные, то вспомогательные задачи будут зависимы от одной переменной.

Ключевые слова: метод Фурье, Maple, дифференцииальное уравнение.
\end{abstract}

\section{Introduction}

Рассмотрим однородное дифференциальное уравнение в частных производных:

$$
L_{x}(u)+M_{t}(u)=0, a<x<b, c<t<d
$$

$(a, b)$ - конечный интервал, $(c, d)$ - конечный или бесконечный интервал, $p(x), p^{\prime}(x), q(x)$, $r(x)$ _ непрерывные функции в $(a, b), L_{x}(u)$,

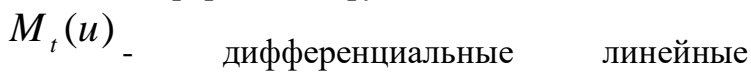
операторы:

$$
L_{x}(u)=\frac{1}{r(x)}\left[\frac{\partial}{\partial x}\left(p(x) \frac{\partial u}{\partial x}\right)-q(x) u\right]
$$

$$
M_{t}(u)=A \frac{\partial^{2} u}{\partial t^{2}}+B \frac{\partial u}{\partial t}+C u
$$

Искомая функция $u=u(x, t)$ по переменной $x$ удовлетворяет одному из граничных условий первого, второго или третьего рода соответственно: 


\begin{tabular}{llllll} 
& ISRA (India) $=\mathbf{3 . 1 1 7}$ & SIS (USA) $=\mathbf{0 . 9 1 2}$ & ICV (Poland) & $\mathbf{= 6 . 6 3 0}$ \\
Impact Factor: & ISI (Dubai, UAE) $=\mathbf{0 . 8 2 9}$ & PUHЦ (Russia) $=\mathbf{0 . 1 5 6}$ & PIF (India) & $=\mathbf{1 . 9 4 0}$ \\
& GIF (Australia) $=\mathbf{0 . 5 6 4}$ & ESJI (KZ) & $\mathbf{8 . 7 1 6}$ & IBI (India) & $=\mathbf{4 . 2 6 0}$ \\
& JIF & $\mathbf{1 . 5 0 0}$ & SJIF (Morocco) $=\mathbf{5 . 6 6 7}$ & OAJI (USA) & $\mathbf{0 . 3 5 0}$ \\
\hline
\end{tabular}

$$
\begin{gathered}
\left.u\right|_{x=a}=0,\left.\quad u\right|_{x=b}=0, \\
\left.\frac{\partial u}{\partial x}\right|_{x=a}=0,\left.\quad \frac{\partial u}{\partial x}\right|_{x=b}=0, \\
\frac{\partial u}{\partial x}-\left.h_{a} u\right|_{x=a}=0, \quad \frac{\partial u}{\partial x}+\left.h_{b} u\right|_{x=b}=0, h_{a}>0, h_{b}>0
\end{gathered}
$$

Также для функции $u=u(x, t)$ выполняются условия по переменной $t$, зависящие от типа уравнения (1). Тип уравнения определяется знаком $A$. Если $A>0$, то (1) уравнение эллиптического типа и на концах интервала $(c, d)$ выполняются условия $(c, d)$ первого, второго, или третьего рода:

$$
\begin{gathered}
\left.u\right|_{t=c}=\varphi_{c}(x),\left.\quad u\right|_{t=d}=\varphi_{d}(x), \\
\left.\frac{\partial u}{\partial x}\right|_{t=c}=\varphi_{c}(x),\left.\quad \frac{\partial u}{\partial x}\right|_{t=d}=\varphi_{d}(x), \\
\frac{\partial u}{\partial x}-\left.h_{c} u\right|_{t=c}=\varphi_{c}(x), \quad \frac{\partial u}{\partial x}+\left.h_{d} u\right|_{t=d}=\varphi_{d}(x) .
\end{gathered}
$$

Уравнение (1) гиперболического типа, если $A<0$. В этом случае, переменная $t$ - время, $t \in(c,+\infty)$ и условия имеют вид

$$
\left.u\right|_{y=c}=\varphi(x),\left.\quad \frac{\partial u}{\partial y}\right|_{y=c}=\psi(x)
$$

Если $A=0$, то (1) - уравнение параболического типа; переменная $t$ - время, $t \in(c,+\infty)$. Тогда условия таковы

$$
\left.u\right|_{t=c}=\varphi(x)
$$

\section{Materials and Methods}

Применяя метод Фурье, решение задачи находится в виде:

$$
u=u(x, t)=X(x) \cdot T(t) .
$$

Для решения данной задачи воспользуемся средствами математического пакета Maple. Рассмотрим методику решения задачи для уравнения теплопроводности с начальными и граничными условиями . Подключаем специальный пакет для решения дифференциальных уравнений в частных производных PDEtools, пакет линейной алгебры linalg : restart; with(PDEtools) : with(linalg) : Вводятся

значения $a_{11}, a_{12}, a_{22}, a_{1}, a_{2}, a_{0}, d$

уравнения, само уравнение, начальные и граничные данные :

restart; with(PDEtools) : with(linalg) :

a11 $:=-\mathrm{a}^{2} ; \mathrm{b}:=0: \mathrm{a} 22:=0 ; \mathrm{a} 1:=0 ; \mathrm{a} 2:=1 ; a 0:=0 ; d:=0 ; a 12:=\frac{b}{2} ; a 21:=a 12 ; 0$

$$
<x, x<l, t>0
$$

PDE1 := a11·diff $(u(x, t), x, x)+2 \cdot a 12 \cdot \operatorname{diff}(u(x, t), x, t)+a 22 \cdot \operatorname{diff}(u(x, t), t, t)+a 1$

$\cdot \operatorname{diff}(u(x, t), x)+a 2 \cdot \operatorname{diff}(u(x, t), t)+a 0 \cdot u(x, t)+\mathrm{d}=0$

init_c $:=u(x, 0)=\operatorname{phi}(x)$;

bound_c $:=u(0, \mathrm{t})=0, u(1, \mathrm{t})=0$;

$\phi:=x \rightarrow x \cdot(l-x)$;

$$
\begin{gathered}
0<x, x<l, 0<t \\
\text { PDE1 }:=-a^{2}\left(\frac{\partial^{2}}{\partial x^{2}} u(x, t)\right)+\frac{\partial}{\partial t} u(x, t)=0 \\
\text { init_c }:=u(x, 0)=\phi(x) \\
\text { bound_c }:=u(0, t)=0, u(l, t)=0 \\
\phi:=x \rightarrow x(l-x)
\end{gathered}
$$

Выполняем разделение переменных: 


\begin{tabular}{|c|c|c|c|c|c|c|}
\hline \multirow{4}{*}{ Impact Factor: } & ISRA (India) & $=3.117$ & SIS (USA) & $=0.912$ & ICV (Poland) & $=6.630$ \\
\hline & ISI (Dubai, UAE & $=0.829$ & РИНЦ (Russia) & $=0.156$ & PIF (India) & $=1.940$ \\
\hline & GIF (Australia) & $=0.564$ & ESJI (KZ) & $=8.716$ & IBI (India) & $=4.260$ \\
\hline & JIF & $=1.500$ & SJIF (Morocco) & $=5.667$ & OAJI (USA) & $=0.350$ \\
\hline
\end{tabular}

res $:=$ pdsolve $(P D E 1, H I N T=F 1(x) \cdot F 2(t))$;

res $1:=o p(1$, res $)$; res $2:=o p(2$, res $)$;

$\operatorname{res} 2[1]$;

$s 1:=\operatorname{op}(1, \operatorname{res} 2[1]) ; s 2:=o p(2, \operatorname{res} 2[1]) ;$

$$
\begin{aligned}
& \text { res : }=(u(x, t)=F 1(x) F 2(t)) \text { \&where }\left[\left\{\frac{\mathrm{d}^{2}}{\mathrm{~d} x^{2}} F 1(x)={ }_{-} c_{1} F 1(x), \frac{\mathrm{d}}{\mathrm{d} t} F 2(t)=a^{2}{ }_{-} c_{1} F 2(t)\right\}\right] \\
& \text { resl } 1:=u(x, t)=F 1(x) F 2(t) \\
& \text { res } 2:=\left[\left\{\frac{\mathrm{d}^{2}}{\mathrm{~d} x^{2}} F 1(x)={ }_{-} c_{1} F 1(x), \frac{\mathrm{d}}{\mathrm{d} t} F 2(t)=a^{2}{ }_{-}{ }_{1} F 2(t)\right\}\right] \\
& \left\{\frac{\mathrm{d}^{2}}{\mathrm{~d} x^{2}} F 1(x)={ }_{-} c_{1} F 1(x), \frac{\mathrm{d}}{\mathrm{d} t} F 2(t)=a^{2}{ }_{-} c_{1} F 2(t)\right\} \\
& s 1:=\frac{\mathrm{d}}{\mathrm{d} t} F 2(t)=a^{2} c_{1} F 2(t) \\
& s 2:=\frac{\mathrm{d}^{2}}{\mathrm{~d} x^{2}} F l(x)=c_{1} F l(x)
\end{aligned}
$$

Получим два обыкновенных

дифференциальных уравнения $s 1$ и $s 2$. Одно

из полученных уравнений $s 2$ с граничными

условиями

$$
u(0, t)=u(l, t)=0 \text { представляет }
$$

задачу Штурма - Лиувилля с однородными условиями по переменной $x$. Находим общее решение этого уравнения и составляем систему однородных условий по граничным условиям:

$\operatorname{PDE} 2:=1 \mathrm{hs}(s 2)+$ lambda $\cdot F 1(x)$;

assume (lambda $>0)$ : dsolve $(\operatorname{PDE} 2, \mathrm{~F} 1(x))$;

$F 1:=$ unapply $(\operatorname{rhs}(\%), x)$;

e1 $:=\mathrm{F} 1(0)=0 ; \mathrm{e} 2:=\mathrm{F} 1(1)=0$;

sist $:=\{\mathrm{e} 1, \mathrm{e} 2\}$;

$$
\begin{gathered}
P D E 2:=\frac{\mathrm{d}^{2}}{\mathrm{~d} x^{2}} F 1(x)+\lambda F 1(x) \\
F 1(x)=_{-} C 1 \sin (\sqrt{\lambda \sim} x)+{ }_{-} C 2 \cos (\sqrt{\lambda \sim} x) \\
F 1:=x \rightarrow_{-} C 1 \sin (\sqrt{\lambda \sim} x)+_{-} C 2 \cos (\sqrt{\lambda \sim} x) \\
e 1:={ }_{-} C 2=0 \\
\text { sist }:=\left\{{ }_{-} C 2=0, C 1 \sin (\sqrt{\lambda \sim} l)+{ }_{-} C 1 \sin (\sqrt{\lambda \sim} l)+{ }_{-} C 2 \cos (\sqrt{\lambda \sim} l)=0\right.
\end{gathered}
$$

Вычисляем определитель полученной

системы sist $:=\{\mathrm{e} 1, \mathrm{e} 2\}$; Затем приравниваем определитель нулю и получим уравнение для нахождения собственных значений:

$A:=\operatorname{linalg}[$ genmatrix $]\left(\right.$ sist,$\left.\left\{\_\mathrm{C} 1,{ }_{-} 2\right\}\right) ;$

Delta $:=$ convert $(\operatorname{linalg}[\operatorname{det}](\bar{A}), \operatorname{trig})$;

EnvAllSolutions $:=$ true;

solve $(\Delta, \lambda) ; \operatorname{indets}(\%) \operatorname{minus}\{1\}$;

$\operatorname{subs}(\%[1]=k, \% \%)$;

ev $:=$ unapply $(\%, k)$

$A:=\operatorname{linalg}[$ genmatrix $]\left(\right.$ sist,$\left.\left\{\_C 1, \_C 2\right\}\right) ;$ Delta $:=\operatorname{convert}(\operatorname{linalg}[\operatorname{det}](A)$, trig $)$; 


\begin{tabular}{|c|c|c|c|c|c|c|}
\hline \multirow{4}{*}{ Impact Factor: } & ISRA (India) & $=3.117$ & SIS (USA) & $=0.912$ & ICV (Poland) & $=6.630$ \\
\hline & ISI (Dubai, UAE & $=0.829$ & РИНЦ (Russia) & $=0.156$ & PIF (India) & $=1.940$ \\
\hline & GIF (Australia) & $=0.564$ & ESJI (KZ) & $=8.716$ & IBI (India) & $=4.260$ \\
\hline & JIF & $=1.500$ & SJIF (Morocco) & $=5.667$ & OAJI (USA) & $=0.350$ \\
\hline
\end{tabular}

$$
\begin{aligned}
& A:=\left[\begin{array}{cc}
0 & 1 \\
\sin (\sqrt{\lambda \sim} l) & \cos (\sqrt{\lambda \sim} l)
\end{array}\right] \\
& \Delta:=-\sin (\sqrt{\lambda \sim} l) \\
& \frac{\pi^{2} Z 1 \sim^{2}}{l^{2}} \\
& \left\{\left\{\_Z 1 \sim\right\}\right\} \\
& \frac{\pi^{2} k^{2}}{l^{2}} \\
& e v:=k \rightarrow \frac{\pi^{2} k^{2}}{l^{2}} \\
& \frac{\pi^{2} Z 1 \sim^{2}}{l^{2}} \\
& \left\{\left\{\_Z 1 \sim\right\}\right\} \\
& \frac{\pi^{2} k^{2}}{l^{2}} \\
& e v:=k \rightarrow \frac{\pi^{2} k^{2}}{l^{2}}
\end{aligned}
$$

Зная собственные значения, находим соответствующие собственные функции:

$F 1:=F 1$; assume $(k$, posint $)$;

subs (lambda $=e v(\mathrm{k}), \mathrm{PDE} 2)$;

dsolve $(\{\%, F 1(0)=0, F 1(1)=0\}, F 1(\mathrm{x}))$;

$\frac{\mathrm{d}^{2}}{\mathrm{~d} x^{2}} F 1(x)+\frac{\pi^{2} k^{2} F 1(x)}{l^{2}}$

$F 1(x)={ }_{-} C 1 \sin \left(\frac{\pi k \sim x}{l}\right)$

Найденные собственные функции нормируем:

$\frac{r h s(\%)}{\operatorname{sqrt}\left(\operatorname{int}\left(\operatorname{rhs}(\%)^{2}, x=0 . . l\right)\right)} ; \operatorname{simplify}(\%$, radical, symbolic $) ;$ ef $:=$ unapply $(\%,(k, x)) ;$

$\frac{-C l \sin \left(\frac{\pi k \sim x}{l}\right) \sqrt{2}}{\sqrt{l-C l^{2}}}$

$\frac{\sin \left(\frac{\pi k \sim x}{l}\right) \sqrt{2}}{\sqrt{l}}$

$e f:=(k \sim, x) \rightarrow \frac{\sin \left(\frac{\pi k \sim x}{l}\right) \sqrt{2}}{\sqrt{l}}$

Таким образом, уравнение $s 2$ решено: найдены собственные значения и нормированные

собственные функции. Находим общее решение уравнения $s 1$ : 


\begin{tabular}{|c|c|c|c|c|c|c|}
\hline \multirow{4}{*}{ Impact Factor: } & ISRA (India) & $=3.117$ & SIS (USA) & $=0.912$ & ICV (Poland) & $=6.630$ \\
\hline & ISI (Dubai, UAE & $=0.829$ & РИНЦ (Russia & $=0.156$ & PIF (India) & $=1.940$ \\
\hline & GIF (Australia) & $=0.564$ & ESJI (KZ) & $=8.716$ & IBI (India) & $=4.260$ \\
\hline & JIF & $=1.500$ & SJIF (Morocco & $=5.667$ & OAJI (USA) & $=0.350$ \\
\hline
\end{tabular}

$e v(k) ; e f(k, x) ; \operatorname{PDE} 3:=1 \mathrm{hs}(s 1)+a^{2} \cdot e v(k) \cdot F 2(t) ;$ dsolve(PDE3, F2 $\left.(t)\right)$;

$$
\begin{gathered}
P D E 3:=\frac{\mathrm{d}}{\mathrm{d} t} F 2(t)+\frac{a^{2} \pi^{2} k^{2} F 2(t)}{l^{2}} \\
F 2(t)={ }_{-} C l \mathrm{e}^{-\frac{a^{2} \pi^{2} k \sim^{2} t}{l^{2}}}
\end{gathered}
$$

Решение исходной задачи находим в виде ряда:

$$
\begin{aligned}
& \operatorname{spr}:=\operatorname{Sum}\left(C(k) \cdot \exp \left(-e v(k) \cdot a^{2} \cdot t\right) \cdot e f(k, x), k=1 . \text { infinity }\right) ; \\
& \operatorname{spr}:=\sum_{k \sim=1}^{\infty} \frac{C(k \sim) \exp \left(-\frac{a^{2} \pi^{2} k \sim^{2} t}{l^{2}}\right) \sin \left(\frac{\pi k \sim x}{l}\right) \sqrt{2}}{\sqrt{l}} .
\end{aligned}
$$

Из начальных условий определяем коэффициенты этого ряда и тогда получим решение задачи:

$$
\begin{aligned}
& C k:=\operatorname{Int}((\operatorname{phi}(x) \cdot e f(k, x)), x=0 . . l) ; \\
& C k:=\operatorname{value}(\%) ; \\
& C k:=\operatorname{unapply}(C k, k) ; \\
& \text { sol }:=\operatorname{spr}
\end{aligned}
$$

$$
\begin{aligned}
& C k:=\int_{0}^{l} \frac{x(l-x) \sin \left(\frac{\pi k \sim x}{l}\right) \sqrt{2}}{\sqrt{l}} \mathrm{~d} x \\
& C k:=\left\{\begin{array}{cl}
-\frac{2 \mathrm{I} \sqrt{2}\left((-1)^{k \sim}-1\right)(-l)^{5 / 2}}{\pi^{3} k \sim^{3}} & l \leq 0 \\
-\frac{2 l^{5 / 2} \sqrt{2}\left((-1)^{k \sim}-1\right)}{\pi^{3} k^{3}} & 0<l
\end{array}\right. \\
& C k:=k \sim \rightarrow \text { piecewise }\left(l \leq 0,-\frac{2 \mathrm{I} \sqrt{2}\left((-1)^{k \sim}-1\right)(-l)^{5 / 2}}{\pi^{3} k^{3}}, 0<l\right. \text {, } \\
& \left.-\frac{2 l^{5 / 2} \sqrt{2}\left((-1)^{k \sim}-1\right)}{\pi^{3} k \sim^{3}}\right) \\
& s o l:=\sum_{k \sim=1}^{\infty} \frac{C(k \sim) \exp \left(-\frac{a^{2} \pi^{2} k \sim^{2} t}{l^{2}}\right) \sin \left(\frac{\pi k \sim x}{l}\right) \sqrt{2}}{\sqrt{l}}
\end{aligned}
$$

\section{Conclusion}

Как видно, большая часть программы связана c нахождением собственных значений и собственных функций уравнения $s 2$, которое вместе с граничными условиями представляет задачу Штурма - Лиувилля: от $\operatorname{PDE} 2:=\operatorname{lhs}(s 2)+$ lambda $F 1(x)$; до ef $:=$ unapply $(\%,(k, x))$; При $\quad$ формировании матрицы коэффициентов для $\quad-C 1,-C 2$, возможно столкнуться с трудностями их определения, описываемые в [11]. В этом случае, имеет смысл воспользоваться результатами решения задачи Штурма - Лиувилля с граничными условиями первого рода [11]. Это же 


\begin{tabular}{|c|c|c|c|c|c|c|}
\hline \multirow{4}{*}{ Impact Factor: } & ISRA (India) & $=3.117$ & SIS (USA) & $=0.912$ & ICV (Poland) & $=6.630$ \\
\hline & ISI (Dubai, UAI & $=0.829$ & РИНЦ (Russia & $=0.156$ & PIF (India) & $=1.940$ \\
\hline & GIF (Australia) & $=0.564$ & ESJI (KZ) & $=8.716$ & IBI (India) & $=4.260$ \\
\hline & JIF & $=1.500$ & SJIF (Morocco & $=5.667$ & OAJI (USA) & $=0.350$ \\
\hline
\end{tabular}

возможно и при граничных условиях второго рода по переменной $x$.

Таким образом, использование однородных задач математической физики, несмотря на то, что инструментарий пакета высоко развит и удобен для применения, требует особых разработок и подходов.

\section{References:}

1. Bitsadze, A. V. (1982). Uravneniya matematicheskoy fiziki. (p.336). Moscow: Nauka.

2. Vladimirov, V. S. (1981). Uravneniya matematicheskoy fiziki. (p.512). Moscow: Nauka.

3. Mikhaylov, V. P. (1983). Differentsial'nye uravneniya s chastnymi proizvodnymi. (p.424). M.: Nauka.

4. Goloskokov, D. P. (2004). Uravneniya matematicheskoy fiziki. Reshenie zadach $\mathrm{V}$ sisteme Maple uchebnik dlya vuzov. (p.539). SPb.: Piter.

5. D'yakonov, V. P. (2006). Maple 9.5/10 v matematike, fizike i obrazovanii. Izd: Piter.

6. Tikhonov, A. N., \& Samarskiy, A. A. (1977). Uravneniya matematicheskoy fiziki. Moscow: Nauka.
7. Aramanovich, I. G., \& Levin, V. I. (1964). Uravneniya matematicheskoy fiziki. Moscow: Nauka.

8. Arsenin, V. Y. (1966). Matematicheskaya fizika. Moscow: Nauka.

9. Lynch, S. (n.d.). Dynamical Systems with Applications using Maple. ISBN 0-8176-4150-5

10. Putz, J. F. (2003). Maple Animation. ISBN 158488-378-2

11. Krahmaleva, U., \& Shevtsov, V. (2019). Analytical solution of the regular problem of the Sturm - Liouville problem in maple environment. ISJ Theoretical \& Applied Science, 04 (72), 595-598. Soi: http://s-oi.org/1.1/TAS-04-72-84

Doi: https://dx.doi.org/10.15863/TAS.2019.04.72.84 